\title{
Application of Microhardness Analysis in Multiple Interface Structure of Recycled Concrete
}

\author{
Li Qianqian ${ }^{1, a}$, Li Qiuyi ${ }^{2, b}$ and Yue Gongbing ${ }^{3, c}$ \\ ${ }^{1}$ Postgraduate, School of Civil Engineering, Qingdao University of Technology, Qingdao, China \\ ${ }^{2}$ Professor, Centre for Durability Studies, Qingdao University of Technology, Qingdao, China \\ ${ }^{3}$ P.h.D., School of Civil Engineering, Qingdao University of Technology, Qingdao, China \\ a Email:457552796@qq.com, ${ }^{b}$ Email: Iqyyxn@163.com, ${ }^{\circ}$ Email:yuegongbing@163.com
}

\begin{abstract}
Keywords: recycled concrete, multiple interface model, microhardness, interface transition zone Abstract. Compared with ordinary concrete, recycled concrete has multiple weak interface structure, which is the main reason for the low performance of recycled concrete. Based on the construction of the recycled concrete multi interface model, the vivtorinox hardness of three kinds of interface structure of recycled concrete was tested by Micro Vickers Hardness Tester, in order to characterize the microstructure characteristics of recycled concrete interfacial transition zone. The results show that the microhardness and the width of the interface transition zone can be accurately measured and analyzed by Micro Vickers Hardness Teste after the existing models are sliced, pre grinded and polished. It can be seen that the Micro Vickers Hardness Tester provides a scientific and effective research method for the study of recycled concrete interfacial transition zone.
\end{abstract}

\section{Introduction}

The interface between hardened cement paste and coarse aggregate in concrete is a kind of porous structure called Interfacial Transition Zone (referred to as ITZ). There is a large amount of $\mathrm{Ca}(\mathrm{OH})_{2}$ enrichment and orientation in the interfacial transition zone, which is the weakest link of concrete, and the influence of interfacial transition zone on the mechanical properties and durability of concrete is significant ${ }^{[1,2,3,4]}$. The interfacial transition zone is an area with complex structure, which is difficult to be distinguished. The factors that affect the composition and structure of the region are very complex. From the aspects of the preparation of concrete and the development of strength, the factors are as follows: side wall effect, micro bleeding effect, dehydration shrinkage, ion migration, deposition and nucleation and so on. Compared with ordinary concrete, recycled concrete has multiple interface structure, which is a more complex multiphase complex. It includes the following three interfaces: the old aggregate - the old slurry interface (LG-LJ interface), the old aggregate - the new slurry interface (LG-XJ interface), and the old paste - the new slurry interface (LJ-XJ interface).

A large number of researchers ${ }^{[5,6,7]}$ use electron probe microanalysis (EPXM), scanning electron microscopy (SEM) and electron spectroscopy (EDXA) method to study the interfacial transition zone between aggregate and cement paste, but this is only from the perspective of micro-facet of the concrete interface to do a qualitative analysis of the transition zone. The micro hardness can reflect the characteristics of the interface transition zone of recycled concrete, including the crystal size, porosity size and the crystal orientation index. Therefore, the micro hardness value and the width of the interface transition zone can be quantitatively analyzed. In this paper, the micro hardness of the interface transition zone is measured by using the micro hardness tester, based on the established multiple interface reconstruction model. The application of micro hardness method in the multiple interface of recycled concrete is discussed quantitatively from the point of view of micro mechanics.

\section{Microhardness Measurement Principle and Slice Preparation}

Microhardness Measurement Principle. Hardness is an important mechanical property index, which reflects the ability of solid materials to resist local plastic deformation. Hardness measurement is a certain shape and size of the hard object (head) with a certain pressure into the material surface, 
and the purpose is to determine the material in the deformation process of the resistance. When the load is greater than $9.81 \mathrm{~N}(1 \mathrm{kgf})$, the hardness is called macro hardness. While the pressure is less than $9.81 \mathrm{~N}(1 \mathrm{kgf})$ hardness is called micro hardness ${ }^{[8]}$. In this paper, vivtorinox hardness was used to characterize the micro hardness of recycled concrete interface. Using HX-1000T digital intelligent micro hardness tester, the load is $50 \mathrm{~g}$. Press the head into the surface of the specimen and hold it for a certain period of time. In the sample surface will appear a positive four pyramid indentation and its bottom is square after the load unload. (as shown in Fig.1)Measure the length of the two diagonal lines and get the average value, then get the $d$. The indentation area $F$ and the ratio of the load to the indentation area were calculated. That is vivtorinox hardness $\mathrm{HV}\left(\mathrm{kgf} / \mathrm{mm}^{2}\right)$ :

Indentation area: $F=d^{2} / 2 \sin \theta$.

Vivtorinox hardness: $H V=\frac{P}{F}=P \times 2 \sin \theta / d^{2}=1.8544 P / d^{2}$.

$P$-Load;

$D$-Diagonal length, um;

$\theta$-Contact angle between indenter and material surface, $\theta=68^{\circ}$.

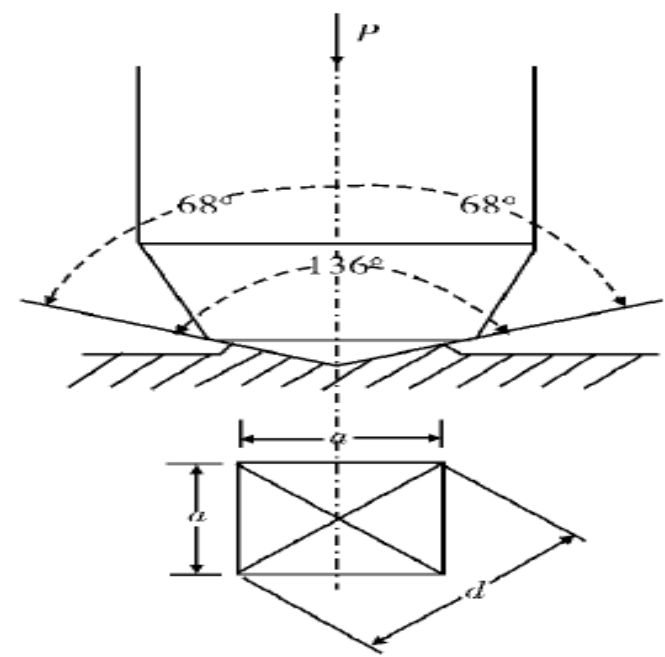

Fig.1. Vivtorinox hardness of diamond pyramid indenter

Preparation of Multi interface Microhardness Slice. Preparation of concrete core sample: Design and make the original coagulation according to the "Design Rules of the Common Concrete Mix".The size of specimen is $800 \mathrm{~mm} \times 600 \mathrm{~mm} \times 100 \mathrm{~mm}$ cuboid. After several months of standard curing, a cylindrical core sample with a diameter of $75 \mathrm{~mm}$ and a height of $100 \mathrm{~mm}$ was drilled.

Slice preparation: Put the pre prepared core sample(soak for 24 hours, decontamination and wipe dry) into the $100 \mathrm{~mm} \times 100 \mathrm{~mm} \times 100 \mathrm{~mm}$ test center, and then pour the cement mortar(as shown in Fig.2). Standard curing to curing age, it was made of $100 \mathrm{~mm} \times 100 \mathrm{~mm} \times 20 \mathrm{~mm}$ slice(as shown in Fig.3). This section contains 3 kinds of interface structure of recycled concrete: LG-LJ interface, LG-XJ interface and LJ-XJ interface. It was immersed in absolute ethanol for 24 hours to terminate hydration. The surface to be measured from the sample, and sandpaper of $320 \mathrm{CW}, 800 \mathrm{CW}, \mathrm{P} 1200$ and P1500 was used to pre-grind in the order of particle size increase by metallographic specimen pre-grinding machine. The samples were pre polished to make the surface smooth and smooth (as shown in Fig.4), so as to meet the requirements of the micro hardness test. 


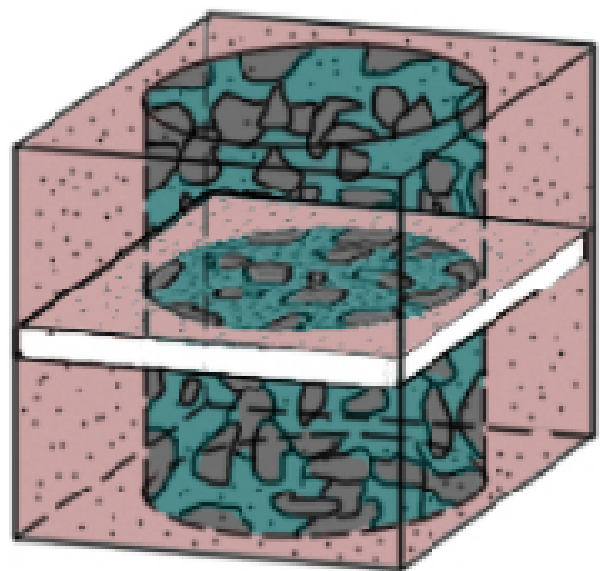

Fig.2. Core sample concrete model

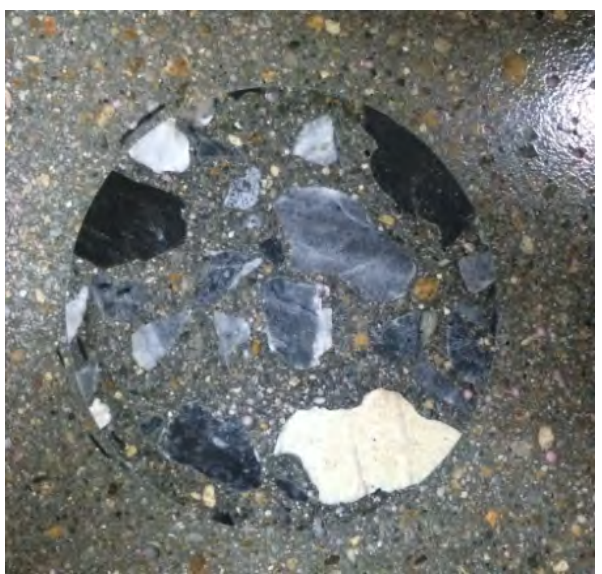

Fig.4. Polished slices

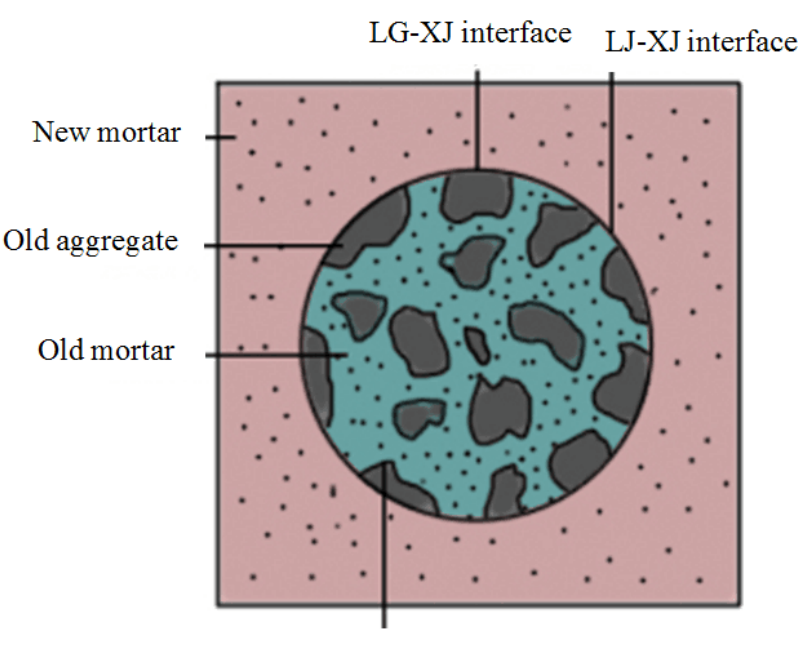

LG-LJ interface

Fig.3. Model of core sample concrete

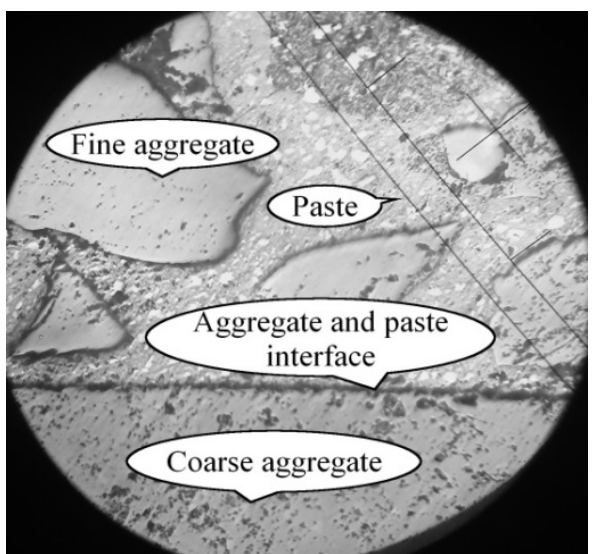

Fig.5.100 times of micro hardness tester interface

Microhardness RBI Way. The interface between LG-XJ and LJ-XJ is similar to that of aggregate and paste in the Fig.5. At $100 \times$, the interface approximates a straight line. The dot pattern is matrix point group, as shown in Fig.6. RBI indentation diagonal length should meet $\mathrm{L} 1>\mathrm{L} 2>20 \mathrm{~mm}$ (reduce visual reading error), and the vertical distance between the two points is $\mathrm{d}>2 \mathrm{~L} 1$ or $50 \mathrm{~mm}$. The lateral distance between two points is $\mathrm{D}$, and the height difference is $10 \mathrm{um}$. Because of the irregularity of the aggregate morphology, the LG-LJ interface in the original concrete core sample presents an irregular curve. So the RBI is a vertical one-way (as shown in Fig.7 and Fig.8).

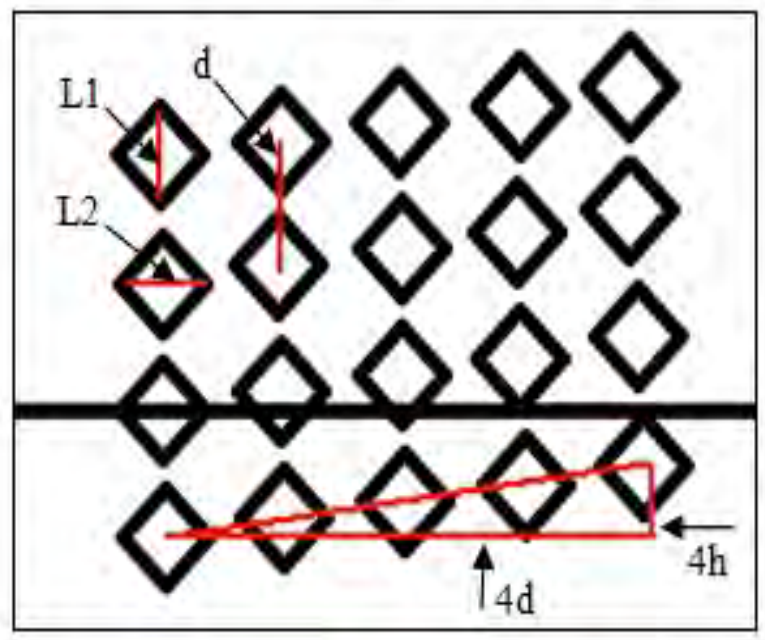

Fig.6. Schematic diagram of micro hardness point group 


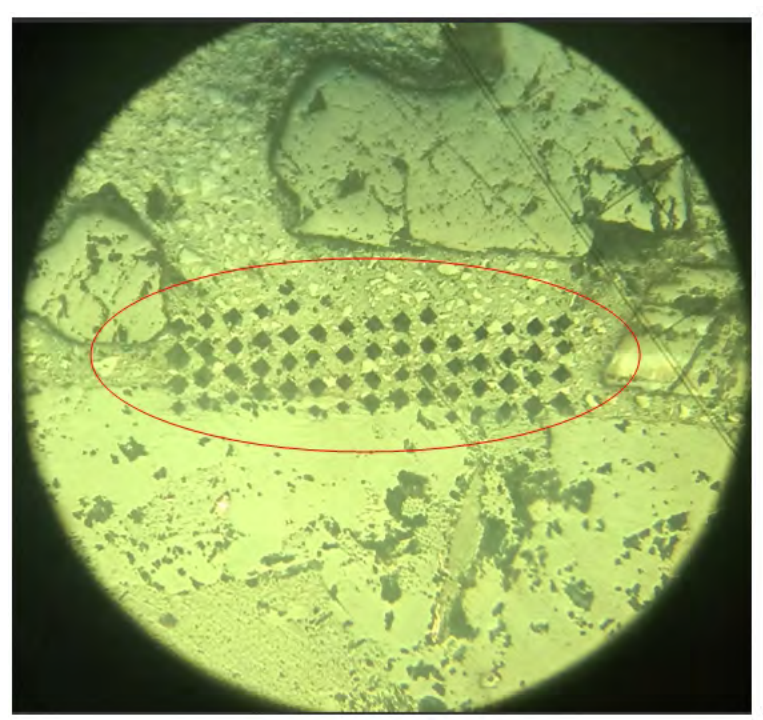

Fig.7. Real point of the matrix group

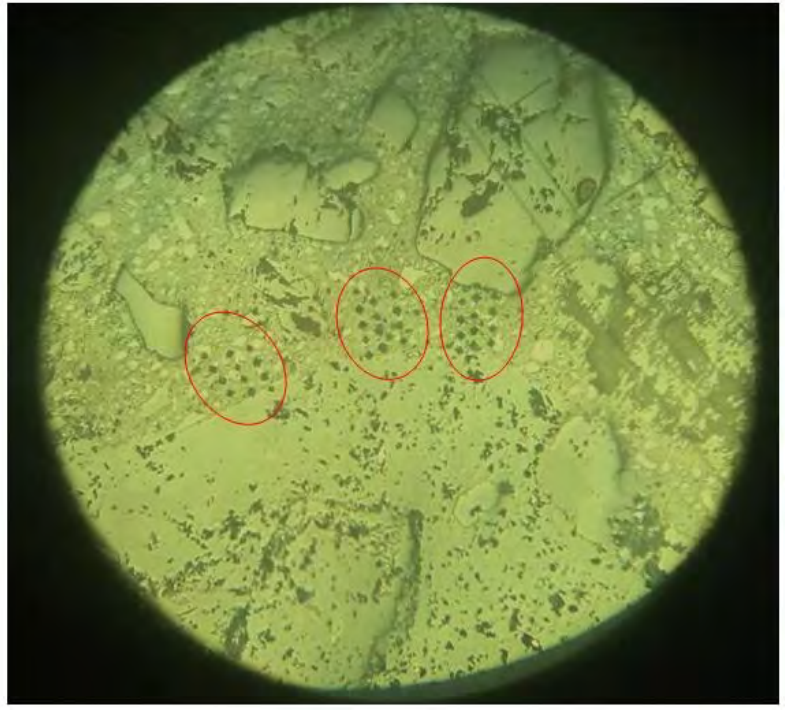

Fig.8. Longitudinal single line pattern

\section{Application Analysis}

Recycled concrete is a more complex multiphase composite. Due to the porosity, surface roughness, aggregate particle distribution and not hydrated clinker particle distribution in recycled concrete, the micro hardness is somewhat discrete. Moreover, in the past, the method of selecting the interface randomly in the recycled concrete to carry out the relevant RBI has a large discrepancy and blindness. Therefore, in the selection of three interfaces for the RBI, the three interfaces should be around the same aggregate in order to reduce the differences in the micro hardness of different aggregate. The influence of various factors makes RBI data strictly not subject to normal distribution. Therefore, we use the box map to deal with the RBI data, with the median as the effective micro-hardness value. At the same time, in order to avoid artificially determining the width of the interface transition zone, a plurality of micro hardness matrix point groups are made in the mortar matrix away from the interface transition zone. The standard values of micro hardness of the mortar matrix were determined by the upper and lower quartiles of the box diagram, as shown in Fig. 10 .

The C40 concrete core samples with standard curing for 6 months were coated with the same strength mortar. After 28 days of standard curing, the micro hardness values of the sections were measured with a micro hardness tester. For the three interfaces in the recycled concrete, there are nine points as shown in Fig. 6 . The points with the same gradient from the interface are processed in box plot, so the micro hardness values at the gradient were obtained. As shown in Fig.9, the box type is the micro hardness value of the LG-LJ interface in the distance of $60 \mu \mathrm{m}$. By analogy, we use the box diagram to process the micro hardness value of each gradient of the interface, so as to obtain a graph of the change in the micro hardness value with the gradient (as shown in Fig.11 (a)). The other two interfaces were processed by the same method to get the curve of the micro hardness with the gradient, as shown in Fig.11 (b) and Fig 11(c). 

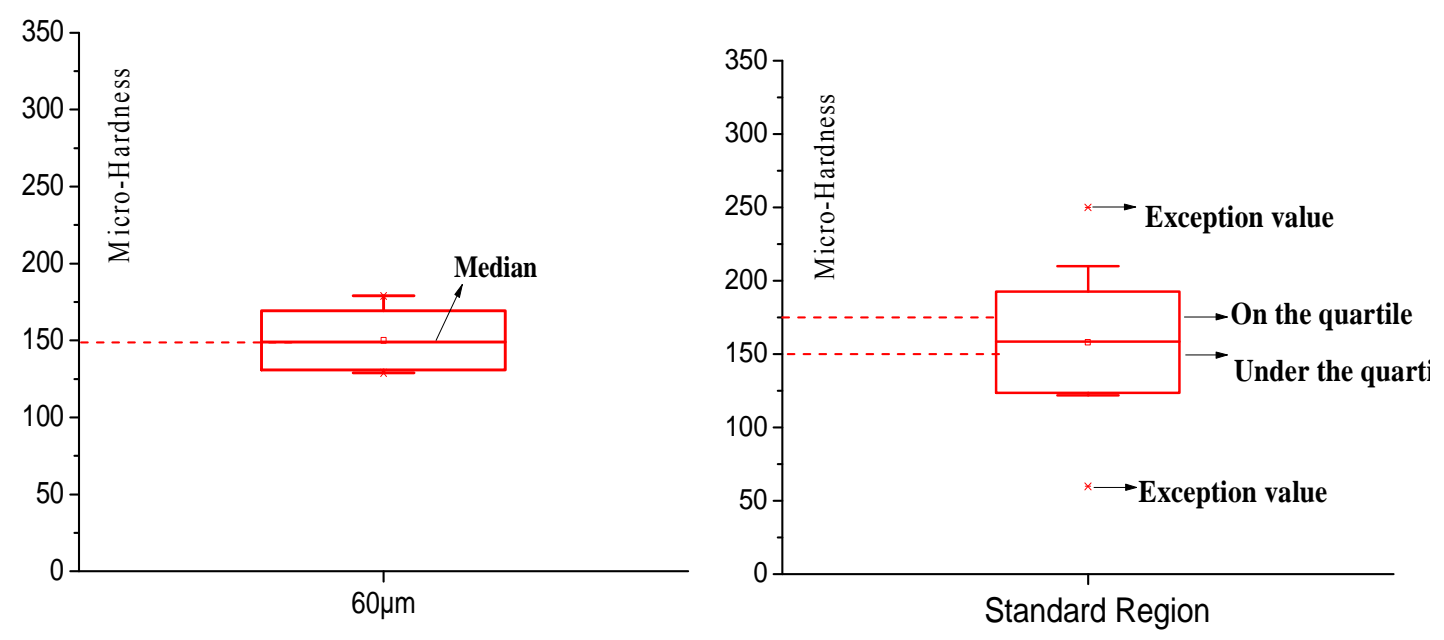

Fig.9 The micro-hardness value of the LG-LJ interface $60 \mu \mathrm{m}$

Fig.10 Standard area box diagram of LG-LJ interface
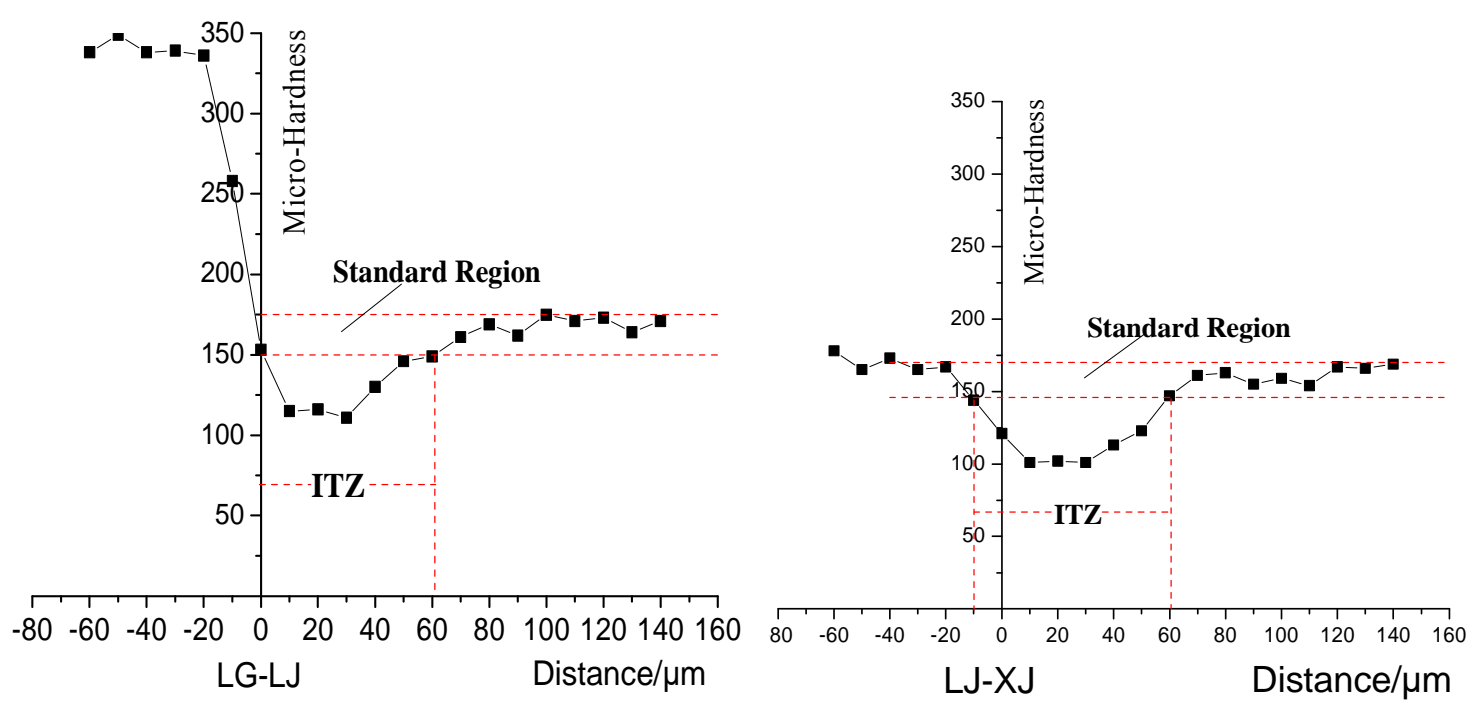

(a) Micro-hardness of LG-LJ interface

(b) Micro-hardness of LJ-XJ interface

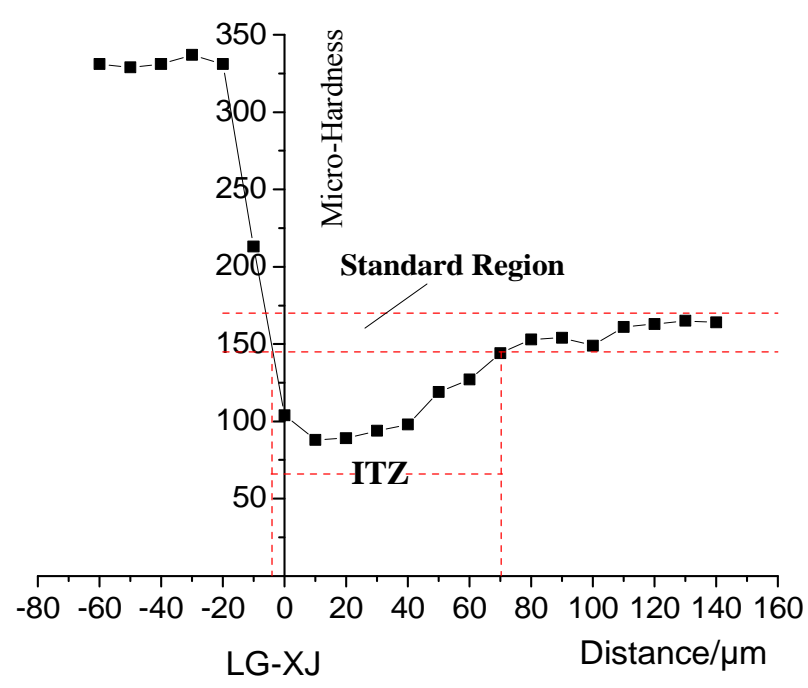

(c) Micro hardness of LG-XJ interface

Fig.11. Schematic diagram of single interface micro hardness

The interfacial transition zone (ITZ) is the area below the standard value of micro hardness. Its width is $H$, the width of the interface transition zone can be seen as follows: $\mathrm{H}(\mathrm{b})>\mathrm{H}(\mathrm{c})>\mathrm{H}$ (a). The 
micro hardness of interfacial transition zone is generally lower than that of the standard region $60 \mathrm{kgf} / \mathrm{mm}^{2}$. As shown in Fig.11 (b) and Fig.11 (c), the micro hardness value at $0 \mathrm{~m}$ is not the lowest value in the whole interface region. This is due to the fact that, at the point of $0 \mathrm{um}$, half of the dot is hit on the aggregate or the old slurry, while the other half is on the new slurry. In addition, the hardness of the points on the old aggregate and the LG-XJ interface in the LJ-XJ interface are also lower than the lower limit of the standard area. This may be due to their ability to be affected by the new slurry to a certain extent, so it is also included in the weak area of the interface structure.

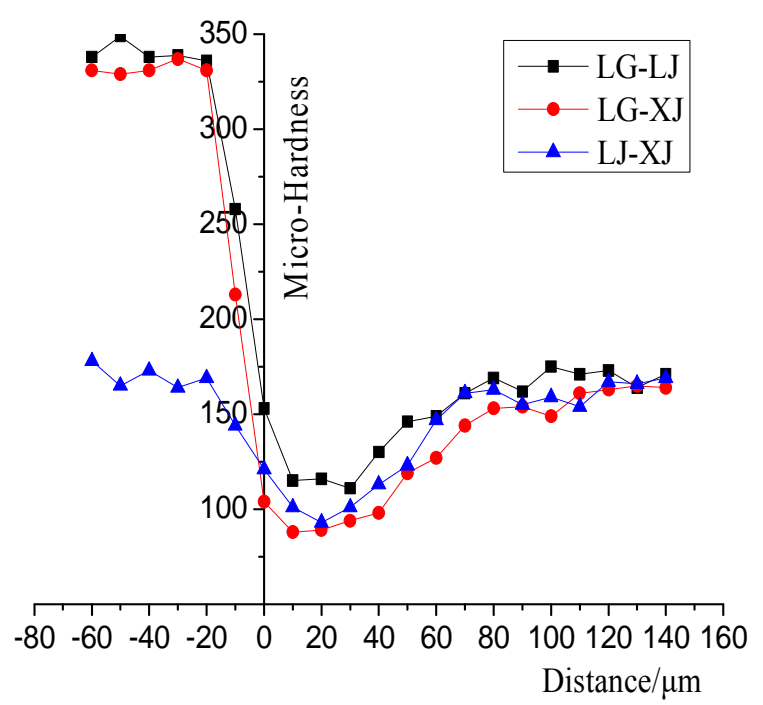

Fig.12. Micro-hardness of interface transition region with $\mathrm{C} 40$

As can be seen from Fig.12, LG-LJ interface, LJ-XJ interface and LG-XJ interface in about 80um away from the interface, a smaller difference between the hardness of their values. In the interface transition zone, the micro hardness of the LG-LJ interface is slightly higher than that of the latter, and the micro hardness of the transition zone is in the order of LG-LJ interface $>$ LJ-XJ interface $>$ LG-XJ. The LG-LJ interface has a high hydration degree and good compactness due to the longer hydration age. However, because of the existence of side wall effect in the LG-XJ interface transition zone, the porosity near the surface of the old aggregate is higher than that of the mortar matrix. This facilitates the migration of water in the slurry and the $\mathrm{Ca}^{2+}, \mathrm{Al}^{3+}$ and $\mathrm{SO}^{2-}$ ions during the hydration process, which result in the enrichment of $\mathrm{Ca}(\mathrm{OH})_{2}$ in the interface transition zone and a low micro hardness of the interface transition zone of LG-XJ. The micro hardness of LJ-XJ interface transition zone is higher than that of LG-XJ interface transition zone, which may be two reasons: First, the roughness of the old slurry surface is larger, so it can form a mosaic occlusion with the new slurry; Secondly, because the water absorption of the old slurry is higher than that of the aggregate, in the initial stage of hydration, the old slurry absorbs part of the enriched water at the interface to form the self-retaining water, which reduces the cracks at the interface due to dehydration shrinkage. At the same time, it also provides water for the hydration reaction of cement at the later stage.

\section{Conclusion}

(1)The interface model of LJ-XJ, LG-XJ and LG-LJ in regenerated concrete can be accurately located and analyzed, which can be used to reveal the failure mechanism of recycled aggregate concrete by using the original concrete core-like method.

(2) The micro hardness tester has the advantages of simplicity and intuition for characterizing the interface transition zone between the aggregate - the slurry and the slurry - the slurry in the recycled concrete. 
(3) At the distance of LJ-XJ interface, LG-XJ interface and LG-LJ interface within 100um, there are obvious interface transition zone. The microhardness of interfacial transition zone is about $60 \mathrm{kgf} / \mathrm{mm}^{2}$ lower than that of the mortar matrix, which can adversely affect the mechanics and durability of recycled concrete.

(4) In the LJ-XJ interface and the LG-XJ interface structure, the new slurry degrades the old slurry and the old aggregate to a certain extent, which reduces the microhardness of the interface transition zone and widens the interfacial transition. Therefore, it is necessary to perform particle shaping of recycled aggregate in the preparation of recycled concrete. We need to remove the mortar attached to the surface of the recycled aggregate, which is subject to durability erosion.

\section{Acknowledgement}

National natural science foundation (No: 51378270).

National natural science foundation (No: 51578297).

\section{References}

[1] Li X P. Recycling and reuse of waste concrete in China: Part II. Structural behaviour of recycled aggregate concrete and engineering applications[J]. Conserv Recy, (2009) 53(3):107-112.

[2] Xiao J Z, Li J B, Mechanical properties of recycled aggregate concrete under uniaxial loading[J]. Cem Concr Compos, (2005) 35(6):1187-1194.

[3] Chen Huisu, Sun Wei, Piet S. Summarization of research on aggregate and slurry interface of cement - based composites (2): Formation of micro - structure at interface - mechanism of deterioration and its influence [J]. Journal of the Chinese Ceramic Society, (2004) 32 (1): 70-79.

[4] Shima H, Tateyashiki H, Matsuhashi R, Yoshida Y. An advanced concrete recycling technology and its applicability assessment through input-output analysis[J].Adv Concr Technol, (2005) 3(1):53-67.

[5] LI Wen-gui, XIAO Jian-zhuang, HUANG Liang, SURENDRA P.SHAH. Experimental study on mechanical properties of interfacial transition zones in recycled aggregate concrete[J].Journal of Hunan University(Natural Sciences),(2014) 41(12):31-39.

[6] HE Zhi-hai, QIAN Chun-xiang, ZHANG Yi, ZHAO Fei. Nanoindentation characteristics of interfacial transition zones in concrete under equal compressive strength[J]. Journal of Building Materials, (2014) 17(6): 1082-1086.

[7] SHI Hui-sheng, SUN Dan-dan, WU Kai. Research progress on microstructure and numerical simulation of interface interfacial transition zone [J].Journal of the Chinese Ceramic Society,(2016) 44(5):678-685.

[8] Lian Li, Yin Haichun, Liao Weidong. Study on microhardness of concrete interface zone [J] Foreign Building Materials Science and Technology, (2005) 26 (2): 8-11. 